\title{
Teaching Fourier optics through ray matrices
}

\author{
I Moreno¹, M M Sánchez-López ${ }^{1}$, C Ferreira², J A Davis ${ }^{3}$ \\ and $\mathrm{F}$ Mateos ${ }^{1}$ \\ ${ }^{1}$ Departamento de Ciencia y Tecnología de Materiales, Universidad Miguel Hernández, \\ E03202 Elche, Spain \\ ${ }^{2}$ Departamento de Óptica, Universidad de Valencia, E45100, Burjassot, Spain \\ ${ }^{3}$ Department of Physics, San Diego State University, San Diego, CA 92182, USA
}

Received 16 November 2004

Published 7 February 2005

Online at stacks.iop.org/EJP/26/261

\begin{abstract}
In this work we examine the use of ray-transfer matrices for teaching and for deriving some topics in a Fourier optics course, exploiting the mathematical simplicity of ray matrices compared to diffraction integrals. A simple analysis of the physical meaning of the elements of the ray matrix provides a fast derivation of the conditions to obtain the optical Fourier transform. We extend this derivation to fractional Fourier transform optical systems, and derive the order of the transform from the ray matrix. Some examples are provided to stress this point of view, both with classical and with graded index lenses. This formulation cannot replace the complete explanation of Fourier optics provided by the wave theory, but it is a complementary tool useful to simplify many aspects of Fourier optics and to relate them to geometrical optics.
\end{abstract}

\section{Introduction}

The education of physicists and engineers in the fields of optics, photonics and imaging is growing in importance because of their multiple applications [1]. Geometrical and Fourier optics are basic topics usually included in optics courses in physics or engineering degrees $[2,3]$. The use of simple matrix algebra has been successfully applied in the study of several optical topics [4] including geometrical systems (ray matrices), polarization optics (Jones matrices) and transmission and reflection properties of thin films and layered media (dynamic matrices). These matrix methods are particularly useful when a large number of elements are considered. Matrix algebra is widely taught in mathematical courses in many scientific and technical degrees and the introduction of optical topics based on this formalism becomes familiar to students.

In this work, we use the ray-matrix formalism for the study and derivation of Fourier optics. Although ray optics does not provide an explanation of the wave phenomena, its simplicity makes it very useful for the analysis of certain aspects of modern optical elements. 
For instance, the stability condition for a laser resonator is usually analysed in terms of ray optics in many textbooks [4-6]. Fourier optics is a subject which exploits the wave nature of light, and therefore it is usually introduced by means of diffraction integrals [7]. However, certain aspects of Fourier optics theory (such as the location of Fourier related planes and the size of the Fourier transform) can be derived in a simple way using ray optics [8]. In addition, ray matrices [9] or equivalent operators [10] have been employed in the description of optical Fourier transform systems.

In this work, we introduce Fourier optics based on the ray-matrix formalism. We use a general factorization of the ray matrix closely related to the Fourier transform, which allows us to derive the Fresnel diffraction equation in a very simple way. In the last decade, the fractional Fourier transform (FRFT) - the extension of the Fourier transform to arbitrary orders-has attracted a lot of interest in the optical community. Here we also present the application of the ray-matrix formalism to analyse FRFT systems. We include several examples with classical refractive and with graded index lenses. Although the proposed analysis cannot replace the complete explanation of Fourier optics provided by wave theory, it is a useful complement to simplify calculations and to relate Fourier and geometrical optics. This formulation represents an easy and compact derivation of Fourier optics, useful for teaching the topic to undergraduate students in physics or engineering degrees.

The outline of the paper is as follows: in section 2 we review the main concepts of raymatrix optics, the matrices of the basic elements and their physical significance. In section 3 we introduce the connection between geometrical optics and Fourier optics, and we find the conditions on the ray matrix to obtain a lens system that performs an optical Fourier transform. We include the analysis of some typical examples studied in texts devoted to Fourier optics. Finally, in section 4 we apply the ray-matrix formalism to the analysis of lens systems that perform a fractional Fourier transform.

\section{Ray-matrix optics}

The ray-matrix formalism applies to centred geometrical optical systems under the paraxial approximation. In this approximation, optical rays are considered to travel close to the optical axis ( $z$-axis in figure 1). A ray crossing a plane $z=z_{0}$ is described with two components, the height $r\left(z_{0}\right)$ and the angle $r^{\prime}\left(z_{0}\right)$ at which it crosses the plane. Since the paraxial approximation indicates that the ray travels close to the $z$-axis, $r^{\prime}$ follows the small angle approximation and can be considered as the slope of the ray $r^{\prime}=\mathrm{d} r / \mathrm{d} z$. For simplicity, here we deal with a one-dimensional geometry $(r-z)$. However, the formulation is directly applicable to usual lens systems because of their circular symmetry around the optical axis, or to anamorphic systems that can be factored into horizontal and vertical components.

An optical system changes the position and the angle of the ray. An input ray with coordinates $\left(r_{1}, r_{1}^{\prime}\right)$ at the input plane is changed to an output ray with coordinates $\left(r_{2}, r_{2}^{\prime}\right)$ at the output plane. In the paraxial approximation, these coordinates can be related in the form of a $2 \times 2$ ray matrix $\mathbf{M}$ as

$$
\left(\begin{array}{l}
r_{2} \\
r_{2}^{\prime}
\end{array}\right)=\left(\begin{array}{ll}
A & B \\
C & D
\end{array}\right)\left(\begin{array}{l}
r_{1} \\
r_{1}^{\prime}
\end{array}\right) .
$$

The most usual application for ray matrices is forming the image of an object. In this case, some important properties of the optical system are obtained when any of the $A B C D$ parameters vanish [10].

- If $A=0$, parallel rays at the input plane are focused at the output plane, which therefore defines the rear or image focal plane of the optical system. 


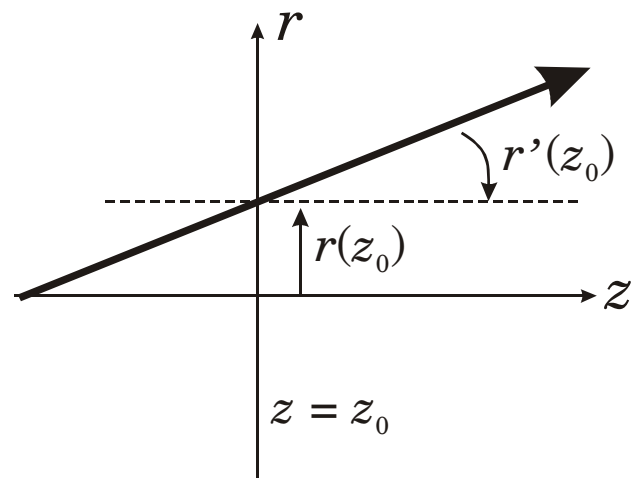

Figure 1. Ray coordinates.

- If $D=0$, a point source at the input plane is converted into parallel rays at the output plane, and therefore the input plane is the front or object focal plane.

- If $B=0$, any point source at the input plane focuses at a corresponding point in the output plane, regardless of the input angle. Therefore, the output plane is the image of the input plane.

- If $C=0$, the system is telescopic. When $C \neq 0$, the system is focal, $C$ being the optical power. The focal length of the system is defined as $f=-1 / C$.

The basic matrices for designing optical systems are the free space propagation of a ray in a homogeneous medium, $\mathbf{M}_{\mathbf{P}}$, and the passage through a spherical refractive thin lens, $\mathbf{M}_{\mathbf{L}}$. These matrices are given by $[3,4]$

$$
\mathbf{M}_{\mathbf{P}}(d)=\left(\begin{array}{ll}
1 & d \\
0 & 1
\end{array}\right)
$$

and

$$
\mathbf{M}_{\mathbf{L}}=\left(\begin{array}{cc}
1 & 0 \\
-1 / f & 1
\end{array}\right),
$$

where $d$ is the distance of propagation and $f$ is the focal length of the thin lens.

Here we also consider the case of selfoc grin media [3-6], which are defined by the inhomogeneous refractive index distribution $n(r)=n_{0} \sqrt{1-\omega^{2} r^{2}}, n_{0}$ being the refractive index on the axis $(r=0)$. The ray trajectories $r(z)$ inside the selfoc grin media are sinusoidal, $\omega$ being the angular frequency of oscillation. The ray matrix describing the selfoc grin media is given by the following expression [4-6]:

$$
\mathbf{M}_{\mathbf{G R I N}}=\left(\begin{array}{ll}
\cos (\omega L) & \frac{1}{\omega} \sin (\omega L) \\
-\omega \sin (\omega L) & \cos (\omega L)
\end{array}\right)
$$

where $L$ is the length of the grin medium. A derivation of these ray matrices can be obtained in many references [3-7].

Because the grin media will be used in section 4 dealing with the fractional Fourier transform, here we analyse them in detail. The distance $L_{\mathrm{p}}=2 \pi / \omega$ is defined as the pitch, and it is the distance corresponding to a complete oscillation of the rays. Depending on the length $L$, a grin lens has different properties, which are summarized in figure 2 . The quarterpitch grin lens $\left(L=L_{\mathrm{p}} / 4\right)$ has a ray matrix with $A=D=0$. Therefore, the front and rear focal planes are located at the input and output planes respectively (figure 2(a)). Rays 

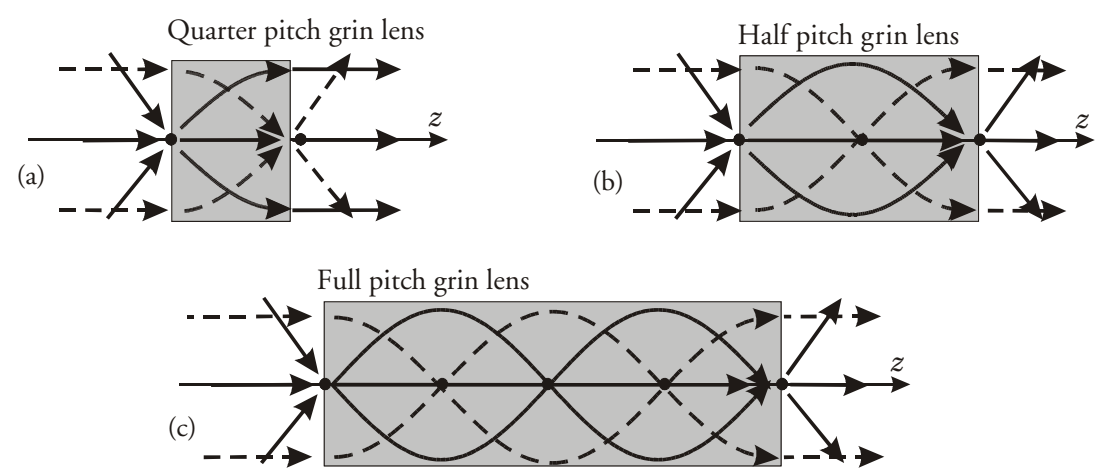

Figure 2. Grin lenses with (a) quarter pitch, (b) half pitch and (c) full pitch.
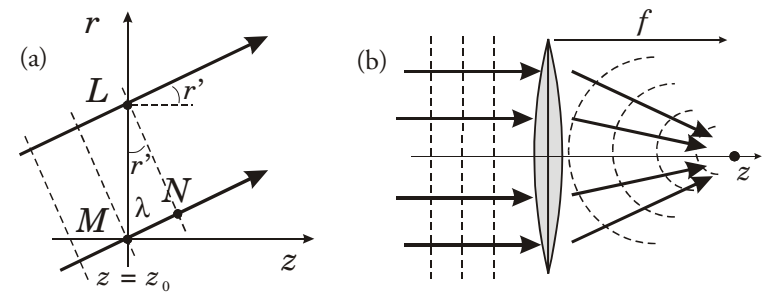

Figure 3. Rays (continuous lines) and wavefronts (dotted lines). (a) A set of parallel rays is equivalent to a linear phase in the plane $r-z$. (b) The effect of a lens is to introduce a quadratic phase factor.

describe a quarter oscillation, so parallel rays are focused on a point while a point source is transformed into a set of parallel rays. The half-pitch grin lens $\left(L=L_{\mathrm{p}} / 2\right)$ has a ray matrix $\mathbf{M}_{\mathrm{GRIN}}=-\mathbf{I}$ where $\mathbf{I}$ is the identity matrix. Because $B=C=0$, the grin lens performs imaging between the input and output planes, while simultaneously acting as a telescopic system. The minus sign indicates the inversion produced in the output coordinates with respect to the input (figure 2(b)). A similar situation occurs for the full-pitch grin lens $\left(L=L_{\mathrm{p}}\right)$, which has a ray matrix $\mathbf{M}_{\mathrm{GRIN}}=\mathbf{I}$. In this case there is no sign inversion (figure 2(c)).

\section{Fourier transform properties and the ray matrix}

The previous formalism is used in many texts to study geometrical optics. However, in general it is not used to teach Fourier transforming optical systems, which are usually treated using diffraction integrals. However, the mathematical simplicity of ray matrices compared to diffraction integrals makes them very useful for this purpose. The connection between ray and wave optics is the correspondence between a set of rays and the corresponding wavefront, which is perpendicular to the ray direction $[8,10]$.

Figure 3 illustrates this connection. We consider a monochromatic illumination with wavelength $\lambda$. A set of tilted parallel rays is equivalent to a tilted plane wavefront, i.e., a linear phase in the plane $z=z_{0}$ (figure 3(a)). The wavefront at the plane $z=z_{0}$ is described as the function $g(r)=\exp [\mathrm{j} 2 \pi r / p]$ where the period $p$ is fixed by the angle $r^{\prime}$. Regarding figure 3(a), points $L$ and $M$ are in phase ( $2 \pi$ phase difference) if the distance $M N$ is equal to the wavelength of the optical radiation. Then, assuming the paraxial approximation, the period $p$ 
is equal to the fraction $\lambda / r^{\prime}$. Therefore, we can conclude that the set of parallel rays crossing the plane $z=z_{0}$ with inclination $r^{\prime}$ is equivalent to a phase distribution $g(r)$ given by

$$
g(r)=\exp \left[\mathrm{j} 2 \pi \frac{r r^{\prime}}{\lambda}\right] \text {. }
$$

In addition, the action of a lens is to transform a plane wave into a spherical wave whose centre is its rear focal point (figure 3(b)). It is well known that the transmittance $t_{l}(r)$ of a lens of focal length $f$ is a quadratic phase factor [7] given by

$$
t_{l}(r)=\exp \left[-\mathrm{j} \frac{\pi r^{2}}{\lambda f}\right],
$$

which causes the change in the wavefront. Equations (5) and (6) provide the relation between ray and wave optics necessary to relate ray matrices to Fourier optics. They will be used in the following to derive the scale of the Fourier transform and the phase distribution at the output plane when a perfect Fourier transform is not obtained.

\subsection{Exact optical Fourier transform $(A=D=0)$}

We want to find the conditions on the ray matrix that lead to a Fourier transform. It is convenient to rewrite the general matrix equation (1) as

$$
\begin{aligned}
& r_{2}=A r_{1}+B r_{1}^{\prime}, \\
& r_{2}^{\prime}=C r_{1}+D r_{1}^{\prime} .
\end{aligned}
$$

We use the two following properties of the generalized Fourier transform [11]:

(1) The Fourier transform of a delta function is a linear phase in the frequency space:

$$
\mathbf{F T}\{\delta(r-a)\}=\exp [-\mathrm{j} 2 \pi a u] .
$$

(2) The Fourier transform of a linear phase is a delta function in the frequency space:

$$
\mathbf{F T}\{\exp [\mathrm{j} 2 \pi r a]\}=\delta(u-a) .
$$

Here $a$ is a constant, $u$ is the spatial frequency and FT stands for the Fourier transform operation, defined as

$$
G(u)=\mathbf{F T}\{g(r)\}=\int_{-\infty}^{+\infty} g(r) \exp [-\mathrm{j} 2 \pi r u] \mathrm{d} r .
$$

In terms of optical radiation, the delta function is equivalent to a point source, while the linear phase corresponds to a tilted set of parallel rays (equation (5)). Consequently, the first condition $(8 a)$ states that a point in the input plane leads to a constant angle in the output plane and can be easily satisfied in equation $(7 b)$ by making the matrix element $D=0$. The second condition $(8 b)$ states that a constant angle in the input plane leads to a point in the output plane, and can be easily satisfied in equation $(7 a)$ by making the matrix element $A=0$.

Therefore, the ray matrix of a Fourier transforming system must have parameters $A=D=0$. The exact Fourier transform is performed between the front and rear focal planes of the optical system. In the usual case of lenses in air, the ray matrix is unimodular $(A D-B C=1)$ and the Fourier transforming system can be written as

$$
\mathbf{M}_{\mathbf{F T}}=\left(\begin{array}{cc}
0 & B \\
-1 / B & 0
\end{array}\right)
$$




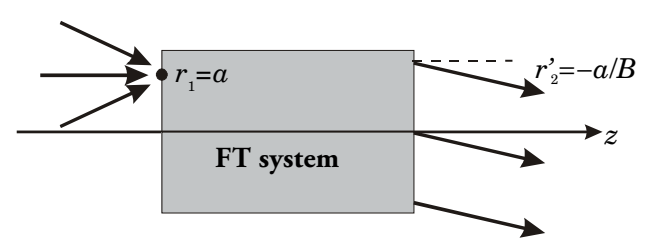

Figure 4. Relation between the location of a point source in the input plane and the angle of the plane wave at the output plane.

where $B$ is equal to the focal length $f_{s}$ of the system. Therefore, an optical system which has a ray matrix in the form of equation (10), produces an optical Fourier transform between its input and output planes. We note that if $D$ is not equal to zero, then the output plane will have a quadratic phase that will not be detected in intensity, as will be shown in section 2.3.

The size of the optical Fourier transform can be easily derived from equation (10). We consider a displaced point source at the input, i.e. $g_{1}\left(r_{1}\right)=\delta\left(r_{1}-a\right)$. Input rays can be considered to have fixed coordinate $r_{1}=a$ and variable angle $r_{1}^{\prime}$ (figure 4). The output rays are given by

$$
\left(\begin{array}{l}
r_{2} \\
r_{2}^{\prime}
\end{array}\right)=\left(\begin{array}{cc}
0 & B \\
-1 / B & 0
\end{array}\right)\left(\begin{array}{l}
a \\
r_{1}^{\prime}
\end{array}\right)=\left(\begin{array}{c}
B r_{1}^{\prime} \\
-a / B
\end{array}\right)
$$

The output angle $r_{2}^{\prime}$ is constant (equal to $-a / B$ ), and therefore they are parallel rays. The equivalent phase distribution at the plane $z=z_{2}$ is given, from equation (5), by

$$
g_{2}\left(r_{2}\right)=\exp \left[\mathrm{j} 2 \pi \frac{r_{2} r_{2}^{\prime}}{\lambda}\right]=\exp \left[-\mathrm{j} 2 \pi \frac{r_{2} a}{\lambda B}\right] .
$$

The comparison of equations (12) and ( $8 a$ ) leads to the relation between the spatial frequency $u$ of the input signal and the spatial coordinate $r_{2}$ at the plane $z=z_{2}$,

$$
u=\frac{r_{2}}{\lambda B}=\frac{r_{2}}{\lambda f_{s}},
$$

where we write $B=f_{s}$, the focal length of the optical system performing the Fourier transform. This equation indicates the size of the Fourier transform through the relation between the spatial coordinate $r_{2}$ at the output plane and the spatial frequency $u$ of the function at the input plane.

Figure 5 shows two simple optical systems that fulfil the Fourier transform condition. The first one is the classical $2 f$ optical system that performs the Fourier transform between the front and rear focal planes of a converging lens. The second one consists of two converging lenses with identical focal length $f$, separated by a distance $d=f$. Both systems perform a Fourier transform between the input plane $P_{1}$ and the output plane $P_{2}$ [8]. In both cases, the ray matrix from $P_{1}$ to $P_{2}$ takes the form of equation (10) with $B$ equal to the focal length $f$. Figure 5 shows the trajectories of some rays, illustrating the Fourier transformation between a point and a plane wave. A third example involves a quarter-pitch grin lens. Its ray matrix also takes the form of equation (10) with $B=1 / \omega$. Figure 2(a) shows the trajectories of some rays illustrating this example.

The above discussion shows that the ray-matrix method provides a simple solution for determining the location of Fourier related planes and the size of the Fourier transform. The specific shape of the wavefront in this plane requires performing the Fourier transform of the input transparency (equation (9)). However, the simple case of a diffraction grating can be very easily analysed in terms of propagation of rays. It is very well known that a diffraction grating illuminated with a collimated plane wave generates plane waves at angles 

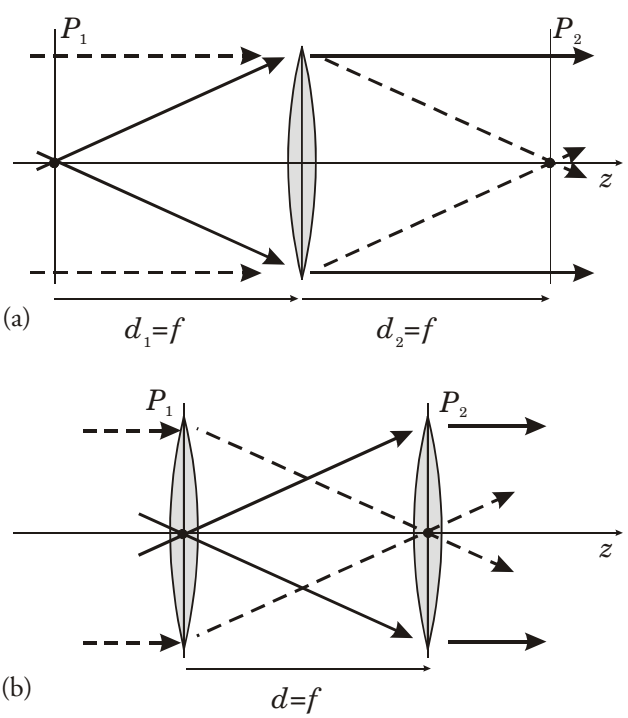

Figure 5. Two lens systems that perform Fourier transform between planes $P_{1}$ and $P_{2}$.

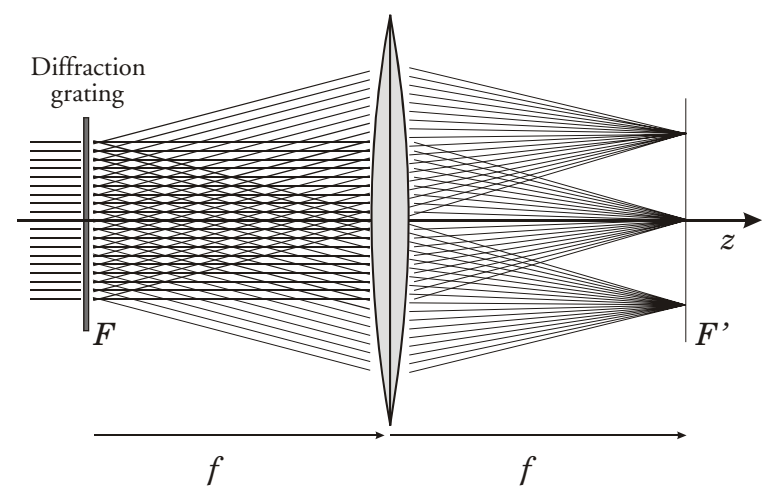

Figure 6. Illustration showing the Fourier transform of a diffraction grating.

of constructive interference given by the law $r_{m}^{\prime} \approx \sin \left(r_{m}^{\prime}\right)=m \lambda / T_{0}$, where $T_{0}$ is the period of the diffraction grating and $m$ is an integer number. Figure 6 shows the trajectories in the $2 f$ Fourier transform system of some rays associated with the plane waves corresponding to $m=0$ and $m= \pm 1$ diffracted orders. The result shows how the rays focus to form the diffraction orders in the Fourier plane.

\subsection{Factorization of a general ray matrix. Application to Fresnel transform}

In this section, we present a factorization of the general ray matrix which provides an easy interpretation in terms of Fourier transforming properties. In [12], a factorization of the ray matrix was proposed related to the principal planes. Here we consider a different factorization proposed in [13] where a general ray matrix is decomposed as

$$
\left(\begin{array}{ll}
A & B \\
C & D
\end{array}\right)=\left(\begin{array}{cc}
1 & 0 \\
D / B & 1
\end{array}\right)\left(\begin{array}{cc}
0 & B \\
-1 / B & 0
\end{array}\right)\left(\begin{array}{cc}
1 & 0 \\
A / B & 1
\end{array}\right) .
$$


This factorization is valid when the input and output planes are not conjugated $(B \neq 0)$. The two matrices in the extremes take the form of thin lenses (equation (3)) while the central matrix takes the form of the Fourier transform system (equation (10)). Therefore a general system can be viewed as a Fourier transform system characterized by a focal length $f=B$, inserted between two lenses with focal lengths $f_{1}=-B / A$ and $f_{2}=-B / D$ at the input and output planes respectively. This decomposition provides a useful connection between the amplitude of the wavefront at the input and output planes, $g_{1}\left(r_{1}\right)$ and $g_{2}\left(r_{2}\right)$ respectively. From the wave-optics point of view, the passage through a lens is equivalent to a multiplication of the wavefront by a quadratic phase factor given by equation (6). The Fourier transform operation is given by equation (9) and its scale is fixed by the parameter $B$ through equation (13). Therefore, applying the decomposition in equation (14), the wavefront amplitude at the output $g_{2}\left(r_{2}\right)$ is obtained, except for a constant factor, by multiplying the input wavefront $g_{1}\left(r_{1}\right)$ by a quadratic phase factor, calculating its Fourier transform, and multiplying the result by another quadratic phase. The results lead to the following relation, known as the generalized diffraction equation or Collins equation [14]:

$$
g_{2}\left(r_{2}\right)=\exp \left[\mathrm{j} \frac{\pi r_{2}^{2} D}{\lambda B}\right] \mathbf{F T}\left\{\exp \left[\mathrm{j} \frac{\pi r_{1}^{2} A}{\lambda B}\right] g_{1}\left(r_{1}\right)\right\}_{u=r_{2} / \lambda B} .
$$

A case of particular interest is the interpretation of Fresnel diffraction. The application of the previous factorization to the free space propagation matrix (equation (2)) leads to

$$
\left(\begin{array}{ll}
1 & d \\
0 & 1
\end{array}\right)=\left(\begin{array}{cc}
1 & 0 \\
1 / d & 1
\end{array}\right)\left(\begin{array}{cc}
0 & d \\
-1 / d & 0
\end{array}\right)\left(\begin{array}{cc}
1 & 0 \\
1 / d & 1
\end{array}\right)
$$

This equation shows that the free space propagation (Fresnel transform) is equivalent to a Fourier transform system, multiplied at both extremes by diverging lenses of focal length $f=-d$. The decomposition in equation (16) directly leads to the very well-known equation for the Fresnel diffraction approximation [7]

$$
g_{2}\left(r_{2}\right)=\exp \left[\mathrm{j} \frac{\pi r_{2}^{2}}{\lambda d}\right] \mathbf{F T}\left\{\exp \left[\mathrm{j} \frac{\pi r_{1}^{2}}{\lambda d}\right] g_{1}\left(r_{1}\right)\right\}_{u=r_{2} / \lambda d} .
$$

The exact Fourier transform system shown in figure 5(b) is obtained by adding compensating converging lenses with focal lengths $f=+d$ on either side of the free propagation.

\subsection{Optical Fourier transform with a phase distribution $(A=0 ; D \neq 0)$}

There exist many other optical architectures where a Fourier transform is obtained, but multiplied by a quadratic phase distribution. They happen when $A=0$ but $D \neq 0$. In this case the factorization in equation (14) directly gives an optical Fourier transform matrix multiplied by a lens with focal length $f=-B / D$, i.e., a quadratic phase factor at the output plane given by equation (6). Often, in practice, we ignore this quadratic phase shift when we detect the output intensity.

As an example we consider a system similar to the $2 f$ shown in figure 5(a), but with the frontal distance between the object and the lens $d \neq f$. This system has a ray matrix

$$
\mathbf{M}=\left(\begin{array}{cc}
0 & f \\
-1 / f & 1-\frac{d}{f}
\end{array}\right) .
$$

Therefore, the output amplitude is the Fourier transform of the input, but it is multiplied by a quadratic phase factor which vanishes only when $d=f$, i.e.,

$$
g_{2}\left(r_{2}\right)=\exp \left[+\mathrm{j} \frac{\pi r_{2}^{2}}{\lambda f}\left(1-\frac{d}{f}\right)\right] \mathbf{F T}\left\{g_{1}\left(r_{1}\right)\right\}_{u=r_{2} / \lambda f} .
$$

This result coincides with those obtained with integral diffraction theory [7]. 


\section{Fractional Fourier transform systems $(A=D)$}

The fractional Fourier transform (FRFT) is the generalization to fractional orders of the Fourier transform operation [15-19]. In the last decade, it has attracted a lot of interest in the optical community since it can be easily obtained by means of optical systems, either with classical lenses [16] or with more versatile programmable diffractive lenses [17].

Optical systems that produce FRFT can also be treated using the ray-matrix formalism [18]. Here we present a simple derivation of the FRFT systems based on the Sylvester theorem [6]. Let us consider an optical elemental system described by a general matrix $\mathbf{M}_{0}$ with parameters $A B C D$. The ray matrix of the $m$-repetition of this elemental system can be calculated by means of the Sylvester theorem [6], which states that

$\mathbf{M}_{0}^{m}=\frac{1}{\sin (\theta)}\left(\begin{array}{cc}A \sin (m \theta)-\sin ((m-1) \theta) & B \sin (m \theta) \\ C \sin (m \theta) & D \sin (m \theta)-\sin ((m-1) \theta)\end{array}\right)$,

where $m$ is an integer and the angle $\theta$ is given by the relation

$$
\cos (\theta)=\frac{1}{2}(A+D) .
$$

If the $m$-repetition of this optical elemental system produces a Fourier transform, the elemental system can be regarded as producing a fractional Fourier transform of order $p=1 / \mathrm{m}$. Therefore, an optical system that produces a FRFT of order $p=1 / m$ must have a ray matrix $\mathbf{M}_{0}$ such that

$$
\mathbf{M}_{0}^{m}=\left(\begin{array}{ll}
A & B \\
C & D
\end{array}\right)^{m}=\left(\begin{array}{cc}
0 & B^{\prime} \\
-1 / B^{\prime} & 0
\end{array}\right),
$$

where $B^{\prime}$ is the parameter characterizing the focal length of the Fourier transform system. By comparing equations (20) and (22), the FRFT condition is satisfied if

$$
A=D=\frac{\sin ((m-1) \theta)}{\sin (m \theta)},
$$

where now $\cos (\theta)=A=D$. These two last relations lead to the condition $\cos (m \theta)=0$, which has the non-trivial solution

$$
\theta=p \frac{\pi}{2}=\frac{\pi}{2 m}
$$

where $p=1 / m$ is the fractional order of the FRFT. Therefore, the ray matrix of an optical system performing a FRFT can be written in the following general form:

$$
\mathbf{M}_{\mathrm{FRFT}}=\left(\begin{array}{cc}
\cos (\theta) & b \sin (\theta) \\
-\frac{1}{b} \sin (\theta) & \cos (\theta)
\end{array}\right),
$$

where the parameter $b$ acts as a scaling factor.

As examples to implement the FRFT we consider the two systems proposed by Lohmann in [16], which are sketched in figure 7. The first system consists of a free propagation of distance $d$, a converging lens of focal length $f$, and a second free propagation of distance $d$. Its ray matrix is

$$
\mathbf{M}_{0}=\left(\begin{array}{cc}
1-\frac{d}{f} & d\left(\frac{2 f-d}{f}\right) \\
-\frac{1}{f} & 1-\frac{d}{f}
\end{array}\right) .
$$

The second system consists of a lens of focal length $f$, a free propagation of distance $d$ and a second lens of the same focal length $f$. In this case the ray matrix is

$$
\mathbf{M}_{0}=\left(\begin{array}{cc}
1-\frac{d}{f} & d \\
-\frac{1}{f}\left(\frac{2 f-d}{f}\right) & 1-\frac{d}{f}
\end{array}\right) .
$$



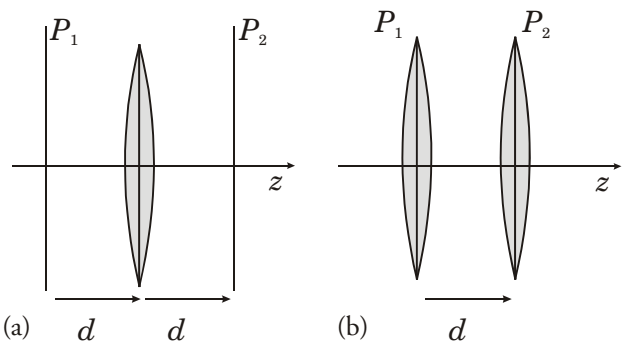

Figure 7. Lohmann lens systems that perform FRFT between planes $P_{1}$ and $P_{2}$. (a) Propagationlens-propagation, (b) lens-propagation-lens.

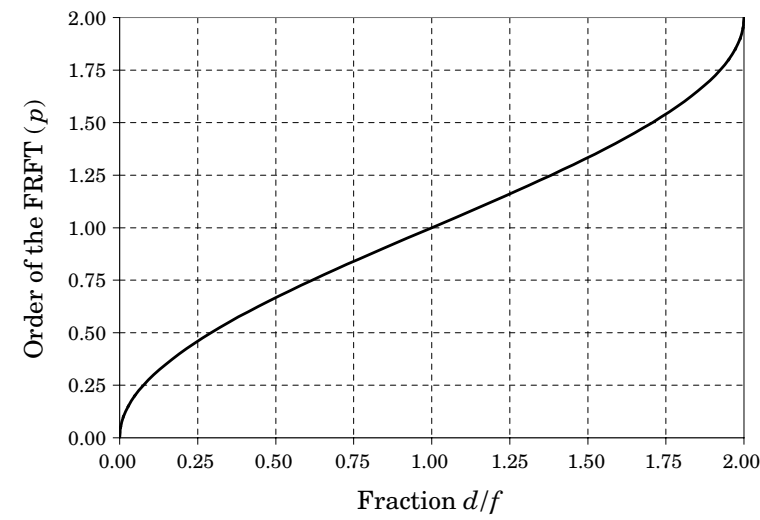

Figure 8. Evolution of the FRFT order $p$ as a function of the fraction $d / f$ in the Lohmann lens systems.

In both cases the ray matrix fulfils $A=D=1-d / f$. Therefore both systems perform a fractional Fourier transform of order $p=2 \theta / \pi$, where $\cos (\theta)=1-d / f$. Consequently, if a FRFT of order $p$ is desired, the relation between $f$ and $d$ is given by

$$
\frac{d}{f}=1-\cos \left(\frac{p \pi}{2}\right)
$$

Again, this equation coincides with the formula derived using diffraction integrals [19]. In both cases, the perfect Fourier transform systems shown in figure 5 are recovered when $d=f$, which corresponds to a FRFT order $p=1$. Figure 8 shows the evolution of the order $p$ as a function of the quotient $d / f$ in the range $p \in[0,2]$, which covers from the image plane to the Fourier transform plane.

A final example of a FRFT system is a grin lens. Early proposals of FRFT systems were based on the propagation in grin media [15]. These properties can be derived very easily using the ray-matrix approach. The ray matrix of a grin lens (equation (4)) follows the FRFT condition through $A=D=\cos (\omega L)$. In this case the angle $\theta$ in equation (25) is equal to $\omega L$. Consequently, a grin lens of length $L$ produces a FRFT of order $p=2 \omega L / \pi$. When $L=\pi / 2 \omega$, i.e., the quarter-pitch grin lens, the Fourier transform is recovered. All these results coincide with those presented in $[15,16]$, and are obtained directly from a very simple analysis of the ray matrix of the optical system. 


\section{Conclusions}

We have presented an analysis of lens systems that perform optical Fourier transforms based on the ray-matrix formalism, as a useful tool for teaching Fourier optics. With this formalism we avoid the use of more complicated diffraction integrals in the resolution of problems such as the location of Fourier related planes, the size of the Fourier transform or the order of a fractional Fourier transform. We have presented a full self-contained derivation of these Fourier optics items based on the ray-matrix formalism, including several examples with refractive lenses or graded index lenses. We have extended this formalism to analyse optical systems that perform fractional Fourier transforms.

\section{Acknowledgments}

This work received support from Ministerio de Ciencia y Tecnología from Spain (projects BFM2003-06273-C02 and FIS2004-06947), and Generalitat Valenciana (project GRUPOS03/117)

\section{References}

[1] 1998 Harnessing Light. Optical Science and Engineering for the 21st century National Research Council, National Academic Press

[2] Yzuel M J 1991 Basic education in optics for physicists Proc. SPIE 1603 109-19

[3] Saleh B E A and Teich M K 1991 Fundamentals of Photonics (New York: Wiley)

[4] Gerrard A and Burch J M 1975 Introduction to Matrix Methods in Optics (New York: Dover)

[5] Siegman A E 1986 Lasers (Mill Valley, CA: University Science Books)

[6] Yariv A 1989 Quantum Electronics 3rd edn (New York: Wiley)

[7] Goodman J 1996 Introduction to Fourier Optics 2nd edn (New York: McGraw-Hill)

[8] Jutamulia J and Asakura T 2002 Optical Fourier-transform theory based on geometrical optics Opt. Eng. 41 $13-6$

[9] Davis J A and Lilly R A 1993 Ray-matrix approach for diffractive optics Appl. Opt. 32 155-8

[10] Nazarathy M and Shamir J 1982 First-order optics-a canonical operator representation: lossless systems J. Opt. Soc. Am. 72 356-64

[11] Bracewell R N 1986 The Fourier Transform and its Applications (New York: McGraw-Hill)

[12] Arsenault H H and Macukow B 1983 Factorization of the transfer matrix for symmetrical optical systems J. Opt. Soc. Am. 73 1350-9

[13] Shamir J and Cohen N 1995 Root and power transformations in optics J. Opt. Soc. Am. A 12 2415-23

[14] Collins S A 1970 Lens-system diffraction integral written in terms of matrix optics J. Opt. Soc. Am. 60 1168-77

[15] Mendlovic D and Ozaktas H M 1993 Fractional Fourier transforms and their optical implementation J. Opt. Soc. Am. A $101875-81$

[16] Lohmann A 1993 Image rotation Wigner rotation, and the fractional Fourier transform J. Opt. Soc. Am. A 10 2181-6

[17] Moreno I, Davis J A and Crabtree K 2003 Fractional Fourier transform optical system with programmable diffractive lenses Appl. Opt. 42 6544-8

[18] Bernardo L M 1996 ABCD matrix formalism of fractional Fourier optics Opt. Eng. 35 740-2

[19] Dorsch R G and Lohmann A 1995 Fractional Fourier transform used for a lens-design problem Appl. Opt. 34 4111-2 